\title{
RETRACTED ARTICLE: NEP 2020: few highlights in higher education
}

\section{Sukanta Kumar Naskar ${ }^{1}$ · Sushovan Chatterjee ${ }^{2} \mathbb{D}$}

Published online: 23 March 2021

(c) Education Research Institute, Seoul National University, Seoul, Korea 2021

The Publisher has retracted this article because it was inadvertently accepted and published owing to an administrative error. The Publisher apologises to the authors and to the Editors-in-Chief. The authors agree with this retraction.
Supplementary Information The online version contains supplementary material available at https://doi.org/10.1007/s12564-021-09668-y.

Sukanta Kumar Naskar

sknaskar@nitttrkol.ac.in

Sushovan Chatterjee

sushovan.chatterjee@gmail.com

1 Department of Education and Management, National Institute of Technical Teachers' Training and Research Kolkata, Kolkata, West Bengal, India

2 Department of Higher Education, Government of West Bengal, Cooch Behar Government Engineering College, Coochbehar, West Bengal, India 